\title{
Defining risk acceptance criteria in occupational settings: A case study in the furniture industrial sector
}

\author{
Matilde A. Rodrigues ${ }^{\mathrm{a}, \mathrm{b}, \text {, , }}$ \\ Pedro M. Arezes ${ }^{\mathrm{b}}$, \\ Celina P. Leão ${ }^{\mathrm{b}}$ \\ ${ }^{a}$ Department of Environmental Health, Research Centre on Environment and Health, \\ Allied Health Sciences School of Polytechnic Institute of Porto, Vila Nova de Gaia, \\ Portugal \\ ${ }^{\mathrm{b}}$ R\&D Centro Algoritmi, Engineering School, University of Minho, Guimarães, Portugal
}

\begin{abstract}
The use of appropriate acceptance criteria in the risk assessment process for occupational accidents is an important issue but often overlooked in the literature, particularly when new risk assessment methods are proposed and discussed. In most cases, there is no information on how or by whom they were defined, or even how companies can adapt them to their own circumstances. Bearing this in mind, this study analysed the problem of the definition of risk acceptance criteria for occupational settings, defining the quantitative acceptance criteria for the specific case study of the Portuguese furniture industrial sector. The key steps to be considered in formulating acceptance criteria were analysed in the literature review. By applying the identified steps, the acceptance criteria for the furniture industrial sector were then defined. The Cumulative Distribution Function (CDF) for the injury statistics of the industrial sector was identified as the maximum tolerable risk level. The acceptable threshold was defined by adjusting the CDF to the Occupational, Safety \& Health (OSH) practitioners' risk acceptance judgement. Adjustments of acceptance criteria to the companies' safety cultures were exemplified by adjusting the Burr distribution parameters. An example of a risk matrix was also used to demonstrate the integration of the defined acceptance criteria into a risk metric. This work has provided substantial contributions to the issue of acceptance criteria for occupational accidents, which may be useful in overcoming the practical difficulties faced by authorities, companies and experts.
\end{abstract}

\section{Keywords}

Acceptance criteria; Decision; Furniture sector; Occupational settings; Risk assessment

\section{Introduction}

Risk assessment is an important process for organizations' safety, allowing them to demonstrate that hazards have been identified, existing risks to worker health and safety 
have been assessed, and measures to reduce risks to a reasonably practicable level have been taken (van Duijne et al., 2008 and CCPS, 2009). However, it is important to recognize that there exist various difficulties during the risk assessment process in the area of occupational accidents, which have been discussed in the literature, such as issues related to the availability of reliable data (Jacinto and Silva, 2010 and Pinto et al., 2012) and the lack of practical tools (Fera and Macchiaroli, 2010 and Pinto et al., 2012). In addition, the appropriateness of the criteria that are considered to support risk decisions is also a problematic question because the use of inappropriate acceptance criteria may result in poor and divergent decisions regarding risk control or mitigation. However, despite its importance, this issue is frequently overlooked when referring to occupational accidents.

Due to the difficulties identified in the risk assessment process, this topic has been the subject of particular attention in recent years. Some researchers have focused their efforts on developing new methodologies and procedures that are, according to the authors, more suitable for application in occupational settings (see e.g. Woodruff, 2005, Marhavilas and Koulouriotis, 2008, Fera and Macchiaroli, 2010, Jacinto and Silva, 2010, Marhavilas et al., 2011 and Carrillo-Castrillo et al., 2014). However, although most of the proposed methods include quantitative criteria presented as acceptance risk limits, the problem of their definition has not been discussed.

In light of the foregoing factors and with the objective of contributing to the discussion regarding the problem of defining risk acceptance criteria for occupational settings, the present study aims to define quantitative acceptance criteria for the specific case of the Portuguese furniture industrial sector via a case study.

The approach and criteria presented in this study do not intend to be an answer to all of the questions related to the decision-making process. Instead, this study intends to address an important problem for the risk assessment process by proposing quantitative criteria for a specific sector, which is useful when defining risk priorities and explaining how they can be defined. Therefore, throughout this study, there is an emphasis on the importance of considering other types of complementary criteria/approaches to support risk decisions.

\subsection{Acceptance criteria as a problematic issue}

Acceptance criteria are terms of reference by which the significance of risk is assessed (ISO Guide 73:2009). In the occupational safety field, different criteria can be used to support decision-making regarding the treatment of risk and setting priorities, as presented by Harms-Ringdahl (2013). However, despite the importance of other criteria, such as requirements of legislation, guidance and good practices (HSE, 2001, Abrahamsen and Aven, 2008 and Harms-Ringdahl, 2013), this study is focused on 
quantitative acceptance criteria, which are materialized as risk limits. The main reason for this specific focus is because most Occupational Safety \& Health (OSH) practitioners use semi-quantitative risk assessment methods to assess the risk of occupational accidents, in which the risk matrix is the principal metric used and the decisions regarding risk acceptance are supported by quantitative criteria (Rodrigues et al., 2012). However, when these types of methodology are used, explanations about the risk criteria used and about who has determined them are relatively scarce (Harms-Ringdahl, 2013). It is not clear whether the criteria used are appropriate or not for the companies' circumstances.

Defining acceptance criteria is not an easy process. According to the Center for Chemical Process Safety (CCPS), organizations have two great challenges when defining acceptance criteria: (i) to ensure appropriate technical accuracy and practical applicability and (ii) to ensure that the risk criteria can be considered credible and equitable (CCPS, 2009). However, some constraints can jeopardize these goals when referring to occupational risks, such as the relatively limited experience and lack of qualified personnel by some companies, as well as the lack of specific guidelines for occupational settings. In fact, the current available guidelines only present general requirements or are more oriented toward major industrial hazards.

\subsection{Model to define risk acceptance criteria in occupational settings}

According to the literature, various important points need to be considered when formulating acceptance criteria (see HSE, 2001, CCPS, 2009, ISO 31000, 2009 and ISO 31010, 2009). The flowchart presented in Fig. 1 schematizes the key steps in defining acceptance criteria for the risk of occupational accidents, taking into account both the guidelines' instructions and the features of the occupational settings. 


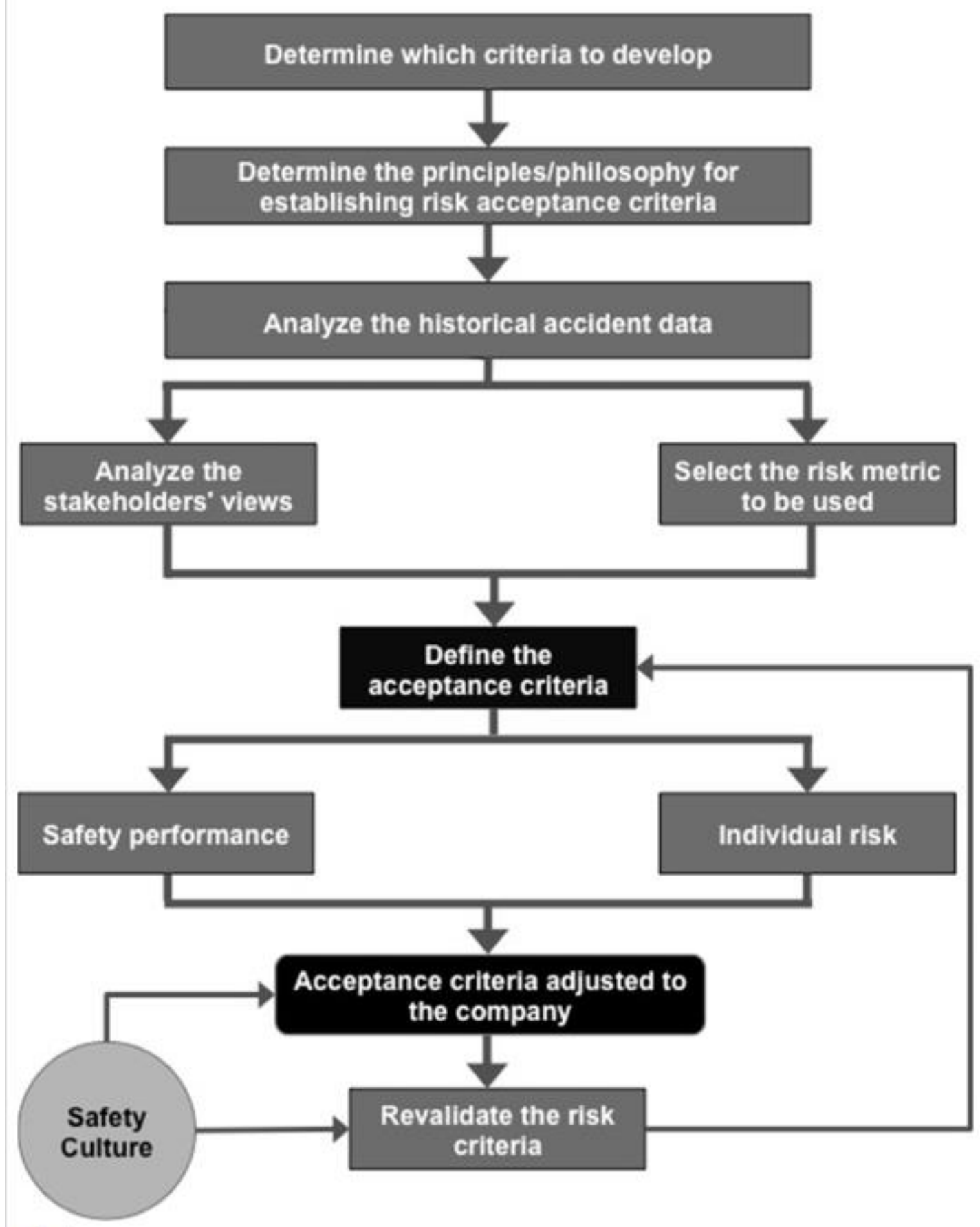

Fig. 1.

Flowchart of the steps to the risk acceptance criteria formulation of occupational accident risks.

A description of each step included in Fig. 1 is given in the following points:

(i)

Determine which criteria to develop: The first step is to determine which criteria are important to establish. Companies with major industrial hazards usually may need to define the acceptance criteria for both individual and societal risks (CCPS, 
2009 and HSE, 2001). Regarding occupational settings, companies usually need to determine the criteria for safety performance and individual risk.

(ii)

Determine the principles/philosophy for establishing risk acceptance criteria: The use of fundamental principles is deeply significant when acceptance criteria are being defined because it can ensure that these criteria are based on rational logic and that they can be easily justified in a transparent manner (Vanem, 2012). Different principles and philosophies for setting risk acceptance criteria can be found in the literature, which can be used alone or together (see e.g. HSE, 2011; Vanem, 2012).

(iii)

Analyse the historical accident data: According to ISO 31000:2009, defining risk criteria requires knowledge about the nature and type of causes and consequences of the accidents that can occur, how they will be measured and how the probability will be defined. This information can be based on the accident databases of the company and/or sector.

(iv)

Analyse the stakeholders' views: It is important to include the stakeholders' judgement about the risk and consider their emotions (Renn, 1997 and ISO 31000, 2009) because of ethical concerns and because they can report useful information (Pidgeon, 1998 and ISO 31000, 2009). Although different stakeholders can be considered, the workers, employers, supervisors and OHS professionals are generally the people who are most interested in a company's safety.

(v)

Select the risk metric to be used: Among many factors (Johansen and Rausand, 2014), the metrics used to estimate the risk level depend on the definition of consequences and likelihood because this selection is limited by the accident dataset available. They are also dependent on the intention of the analysis, i.e. the risk assessment of particular risks or safety performance. This is an important piece of information to consider because acceptance criteria and risk should be expressed on the same scale (Kjellén and Sklet, 1995).

(vi)

Define the acceptance criteria and their adjustment to the company: Based on the outcome of the previous steps, the acceptance criteria can be specified. However, in accordance with ISO 31000:2009, acceptance criteria must be aligned with the organization's safety culture.

(vii)

Periodically revalidate the risk criteria: It is important to consider that risk criteria are dynamic (CCPS, 2009). Therefore, they should be periodically and 
continuously revalidated because the stakeholders' judgement and the companies' goals may change over time.

(viii)

Safety culture: The safety culture can be considered as "the collective ability to produce organizational and interorganizational work practices that protect both individual welfare and the environment" (Tharaldsen et al., 2008). According to this concept, companies with a greater safety culture are expected to demonstrate higher safety performance. Consequently, stricter risk acceptance criteria are required for companies with higher levels of safety culture.

\section{Methodology}

\subsection{Procedures}

Following the steps presented in Fig. 1, quantitative acceptance criteria to be used in assessing the risk of occupational accidents in the furniture industrial sector were developed. First, decisions were made regarding the acceptance criteria to be developed and the principles/philosophy to apply (steps (i) and (ii)). Subsequently, the dataset of work accidents in the furniture industrial sector was analysed (step (iii)). Afterwards, OSH practitioners' views regarding risk acceptance levels were analysed (step (iv)), and the risk metric to be used was selected (step (v)). Based on the results of the previous steps, the acceptance criteria for the furniture industrial sector were finally defined (step (vi)). After finding the acceptance criteria for the entire sector, examples were given of how they can be adjusted to companies' safety cultures and integrated with a risk metric.

\subsection{Accident reports}

Accident reports for the subsector of wood and mattress manufacturing (Code 310 of the Classification of Economic Activities) were used in this survey. The data were supplied by the Portuguese Office of Strategy and Planning (GEP) for the purposes of this study and were derived from the accidents reported by companies. These statistics were grouped by mode of injury according to the European Statistics on Accidents at Work Methodology (ESAW-III) classification (Eurostat, 2013).

\subsection{Analysis of OSH practitioners' risk acceptance}

\subsubsection{Sample}

The study included 1775 Portuguese OSH certified practitioners available in the database of the Portuguese Authority for Work Conditions, who were requested to participate in the study by completing a questionnaire. A total of 271 practitioners replied to the questionnaire, of which only 147 were fully completed. For the present study, all of the 
analyses were performed by considering only the complete questionnaires. This procedure was adopted because the reasons for various questions being left unanswered were unknown and because many of the incomplete respondents did not fill in the most important questions, i.e. the risk scenarios.

Most participants were OSH managers (88.4\%), and 25.9\% had performed their job duties for five years or less, $46.3 \%$ for between 6 and 10 years, $19.7 \%$ for between 11 and 15 years and $8.2 \%$ for more than 16 years. Regarding the type of OSH service provided, $53.1 \%$ of the respondents were in-house OSH personnel, $44.9 \%$ were external consultants, and $2 \%$ provided inter-company services. Only $41.5 \%$ of the respondents worked directly with furniture companies.

\subsubsection{Analysis of Risk Acceptance in the Furniture industrial sector (ARAF) questionnaire}

A questionnaire designated by Risk Acceptance in the Furniture industrial sector (ARAF) was developed and applied.

The preliminary ARAF version was delivered to eight OSH experts. All of them were $\mathrm{OSH}$ managers and were considered experts due to their experience, high level of knowledge and technical competences focused on the risk assessment of occupational accidents (Burgman et al., 2006). The experts examined and tested the questionnaire. Some improvements regarding the language used to describe the scenarios and the scales were suggested and considered in the final version. They were also asked to respond to the questionnaire twice in order to analyse the intra-rater reliability. For scenarios with lower frequencies, the pairwise $k$-coefficients were higher than 0.61 , indicating substantial agreement, while, for scenarios with higher accident frequency, the $k$-values were above 0.81, indicating almost perfect agreement ( Mohan et al., 2000).

The final ARAF version included two main parts. The first part referred to professional characterization, which incorporated four questions. These questions were related to OSH qualification, experience with OSH activities, type of OSH service provided, and whether the respondent works with the furniture industry in his/her professional activities. The second part included 15 questions, each of which was constituted by a set of risk scenarios. A total of 79 scenarios were created. Each one accounted for the frequency of an accident with more lost workdays than a given magnitude. To define the scenarios, different accident frequencies were contemplated for each severity magnitude considering that they must be close to the sector's accident distribution. Different severity magnitudes were analysed: five lost workdays, 25 lost workdays and, after that, intervals of 25 up to 350 lost workdays. The OSH practitioners were requested to classify them as "Acceptable," "Tolerable" or "Unacceptable." 
The request to complete the questionnaire was sent by email, and the questionnaire remained available online for two months.

\subsection{Acceptance criteria development and their integration into a risk metric}

Acceptance criteria for the furniture sector were developed based on the injury distribution for the last year available in the GEP for this study, as well as on the judgement regarding risk acceptance level. To achieve the risk acceptance limits, adjustments to the accident distribution were made in order to approximate it to the OSH practitioners' acceptance levels. Judgements of other stakeholders resulting from previous studies were also considered in the final decision regarding acceptance boundaries.

A risk matrix was selected as the risk metric to be used. The motivation to use this metric/tool was derived from previous studies' results, which showed the risk matrix as being the most frequent measure used by OSH practitioners (Rodrigues et al., 2012). Because the data available were presented as the frequency of accidents by injury severity measured in lost workdays, in the developed risk matrix the "frequency" of accidents was used to give an estimate of the probability of occurrence, whereas the "severity" was established by the corresponding lost workdays. After defining the risk matrix format, the developed acceptance criteria were integrated with it.

\subsection{Data analysis}

Because the accident statistics provided were grouped by mode of injury, it was necessary to extract them into a database to perform the data analysis. Then, to find the distribution of the accidents with injury, the standard distributions, such as Beta, Gamma, Lognormal and Burr, were analysed. The CFD was determined for the distribution with the best fit. The statistical analyses were performed using MATLAB version R2013a and the Microsoft Excel software package.

For OSH professionals' risk acceptance, using the statistical software package IBM SPSS Statistics version 20, a two-step cluster analysis was used to identify the groups of decision-makers. This type of cluster analysis is considered the most appropriate for notably large data files and can be used with both continuous and categorical variables (Shih et al., 2010 and Papadimitriou et al., 2013). It is also given as a good approach when categorical variables with three or more levels are involved (Satish and Bharadhwaj, 2010). It is important to note that the cases were created randomly because the final solution could depend on the order of the cases. 


\section{Results and discussion}

\subsection{Determination of the acceptance criteria to be defined (Step (i))}

It was decided to define the acceptance criteria to be used in the risk assessment of a specific undesired event through the use of aggregated accident data. This was because some small- and medium-sized enterprises (SMEs) lack sufficient accident data to quantify individual risks (Carrillo-Castrillo et al., 2014), and the use of aggregated data has been suggested to reduce subjective judgements and provide a better "picture" of the risk of accidents in relation to specific risk situations, which is useful in defining risk priorities (see e.g. Cuny and Lejeune, 2003, Jacinto and Silva, 2010 and Carrillo-Castrillo et al., 2014).

\subsection{Determination of the principles/philosophy to consider (Step (ii))}

In this case study, the "as low as reasonably practicable" (ALARP) principle was selected. This principle is usually applied with a limit for intolerable risk and a limit for acceptable risk. Accordingly, two risk criteria needed to be defined: (1) an upper risk criterion, which defines the maximum tolerable risk level, and (2) a lower risk criterion, which defines the broadly acceptable risk level.

\subsection{The accident profile of the Portuguese furniture industrial sector (Step (iii))}

By summarizing the main statistical indicators for the Portuguese furniture industrial sector in 2010 (the last dataset available from GEP), it is possible to state that the subsector of wood and mattress manufacturing employed 31,134 workers and recorded 4578 accidents, two of which were fatal. Considering that injuries are the main consequences of accidents in occupational settings, their distribution was analysed, considering previous studies (Cuny and Lejeune, 1999, Cuny and Lejeune, 2003 and Coleman and Kerkering, 2007).

Different continuous distribution models were examined to find the most suitable one for the data. The Beta and the Lognormal distribution models are the most commonly cited models in the literature for occupational accidents (Cuny and Lejeune, 1999, Coleman and Kerkering, 2007 and Sari et al., 2009). However, for this study, the Burr distribution ( $k=0.51866, \alpha=2.1083, \beta=12.634$ ) gave the best fit for the furniture industrial sector injury statistics $(\mathrm{K}-\mathrm{S}=0.04512, p>0.05)$ ( Fig. 2). These results can be related to the large amount of data analysed, as well as the features of the accidents in this sector. In fact, the Burr distribution can fit a wide range of empirical data, and the different values of its parameters cover a wide range of skewness and kurtosis. Furthermore, it has been described as flexible and important in analyses of lifetime and survival data ( Al-Saiari et al., 2014), which also indicates its applicability to occupational accidents. 


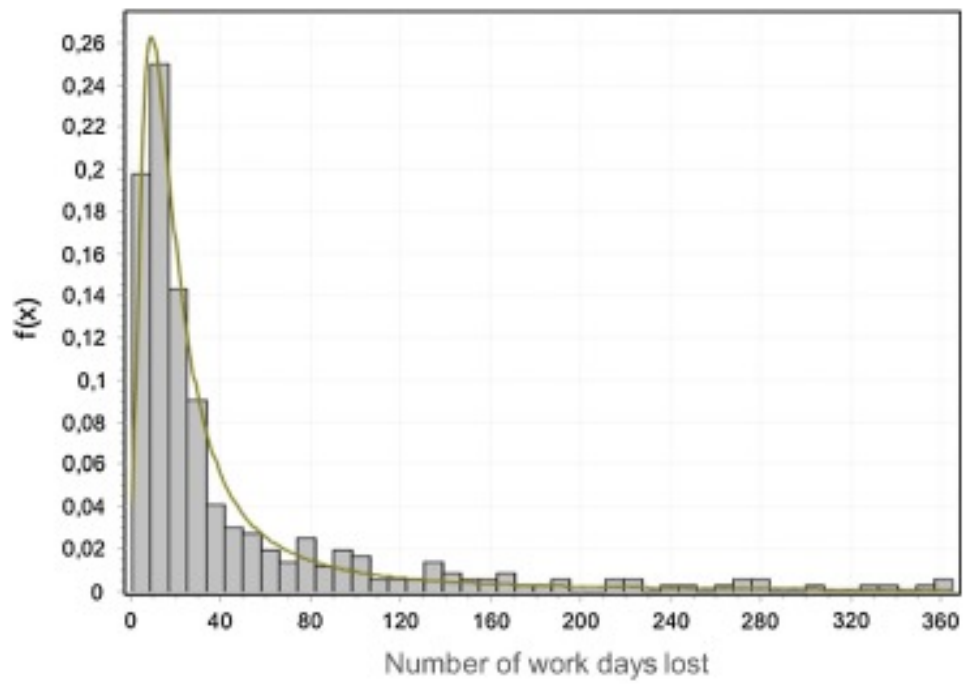

Fig. 2.

Probability density function, $f(x)$, for the number of work days lost.

Finally, the CDF was determined and is presented in Fig. 3. For convenience, when representing the probability of a loss exceeding a given value, the form $1-F(x)$ was used for plotting. This distribution represents the risk curve, in which probability is plotted against severity (number of lost days). In this distribution, the value plotted to a given magnitude of loss represents the probability that a case has more lost days than the given magnitude.

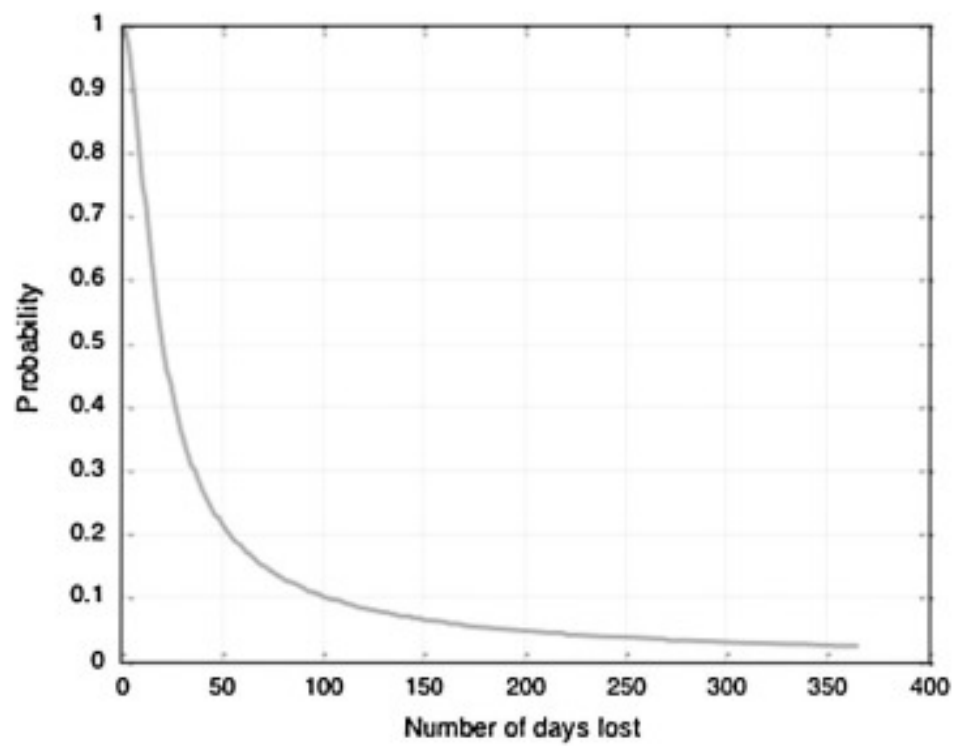

Fig. 3.

CFD for wood and mattress manufacturing injuries. 


\subsection{Analysis of OSH practitioners' views (Step (iv))}

OSH practitioners' judgements regarding risk acceptance levels were analysed because they are more likely to have knowledge about hazards and to conduct quantitative risk assessments than other stakeholders (Siegrist and Cvetkovich, 2000 and Lima, 2005). These judgements were an important consideration in defining the risk acceptance criteria in this study, as presented in Section 3.5. Despite the significance of the results obtained, it is also important to acknowledge their limitations, such as the fact that the initial intention was to include the OSH practitioners that work for the furniture companies analysed. However, it was observed that most Portuguese furniture companies only have external OSH consultants. This situation required an extension to other OSH practitioners.

For the analysis of OSH practitioner judgements, the data collected from the ARAFs were analysed using a two-step cluster analysis. This technique was used to identify groups of decision-makers amongst the OSH practitioners. All respondents that assessed the scenarios with an identical value were neglected for further analysis because the respondents were expected to assess the presented scenarios differently. Accordingly, 29 respondents were removed from the sample.

The cluster analysis was performed for 118 respondents under the following conditions: log-likelihood was used as the distance measure, the confidence interval was 95\%, and the clustering criterion was the Schwarz's Bayesian Criterion. Based on these criteria, three cluster solutions were obtained: (i) the first cluster consists of the decision-makers with negative risk acceptance judgements. This cluster is called the "unacceptable group" because most scenarios were classified as unacceptable and only four scenarios were classified by them as tolerable; (ii) the second cluster, called the "tolerant group," consists of the tolerant decision-makers, of which most classified the scenarios with low risk levels as being tolerable, others as unacceptable and only one as acceptable; (iii) the last cluster consists of the decision-makers with more realistic judgements, and it is called the "realistic group" because their answers show a higher consistency in classifying the scenarios with low risk levels as acceptable, the scenarios with medium risk as tolerable and the scenarios with high risk as unacceptable. The influence of the other variables, such as the OSH professionals' activity, number of years of experience, type of service provided and professional connection to furniture industry companies, were not considered important for the clustering.

The differences observed between the three clusters can be linked to differences in risk perception, trust and emotions, as well as to differences in the safety goals and expectations of OSH practitioners in relation to the occurrence of accidents (Boholm, 1998, Cameron and Raman, 2005, Huijts et al., 2012 and Rodrigues et al., 2015a). For the two first clusters, negative judgements can occur because of the OSH practitioners' 
difficulty in accepting risk scenarios. This situation can result from feelings of distrust, fear, powerlessness and vulnerability, as well as a high level of risk perception (Cameron and Raman, 2005 and Huijts et al., 2012). Furthermore, in some organizations, there is a goal to achieve a target of zero accidents, which may have led these groups to have negative judgements in relation to the scenarios presented. However, it is consensual in the literature that a zero risk level is unattainable (Manuele and Main, 2002 and Hartford, 2009), particularly in some types of industries, such as the furniture industrial sector, where there is a diverse range of risky situations (Miguel et al., 2005). Therefore, it is unrealistic to expect that accidents will not occur in these companies. Consequently, the third cluster was used as the baseline for the acceptance criterion definition because of the consideration of the three risk acceptance levels. The results are presented in Fig. 4.

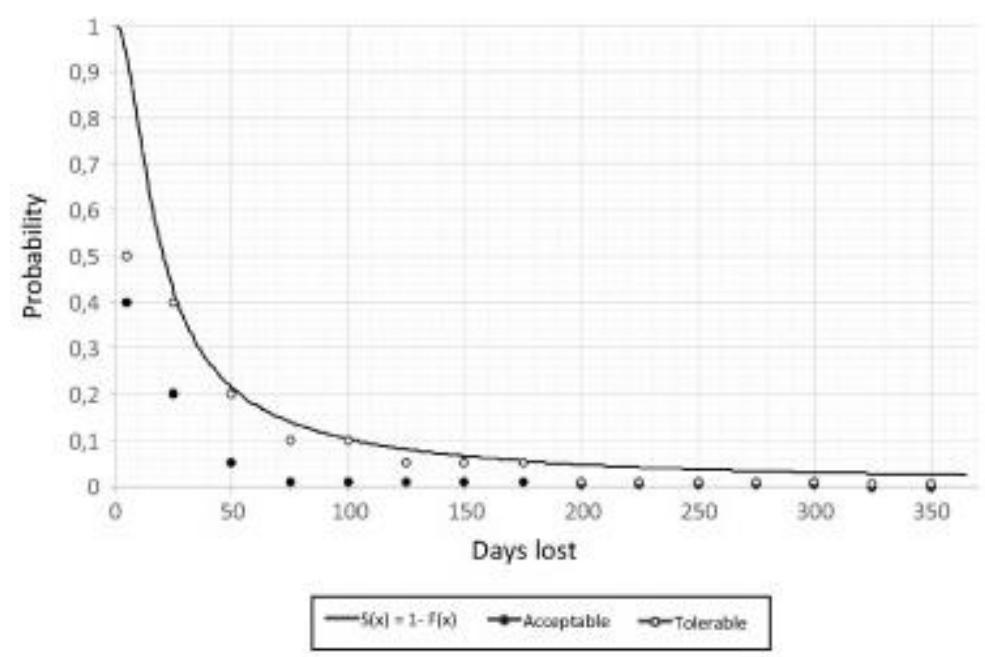

Fig. 4 .

Acceptance risk levels by the "realistic" cluster of OHS practitioners.

Fig. 4 shows that the OHS practitioners identified the maximum limit of tolerable risk close to the CDF curve, $S(x)=1-F(x)$, for most of the situations analysed. A notable exception is the case of a severity of five lost days (the leftmost points on the chart), where a probability of 0.5 was considered tolerable and any value above this limit was considered unacceptable. This number suggests that the OSH practitioners consider more than $50 \%$ of the accidents with a severity equal to or higher than five lost days to be unacceptable. However, this limit is much lower than the statistical distribution of accidents observed in this sector, where more than $90 \%$ of accidents with injuries have a severity of more than five lost days (CDF curve). When the severity is higher than 200 lost days, the maximum tolerable and acceptable levels tend to be close but generally below the CDF curve. This result indicates that they consider high-risk situations as unacceptable, unless they have a notably low probability. 


\subsection{Definition of acceptance criteria and integration into the risk metrics (Steps (v) and (vi))}

Acceptance criteria for the furniture industry were defined by adjusting the CDF curve to the judgements regarding risk acceptance level by OSH practitioners. The results are presented in Fig. 5. Because the limit of tolerable risk identified by the OSH practitioners is notably close to the CDF curve for most situations analysed (Fig. 4), this curve can be used as the maximum tolerable risk level (upper risk criterion). The acceptable threshold (lower risk criterion) was created by adjusting the Burr distribution parameters. Different multiplicative factors were used with the original Burr parameters to adjust the CDF curve to the OSH professionals' risk acceptance levels $(k=0.1297, \alpha=8.4332, \beta=3.1580)$.

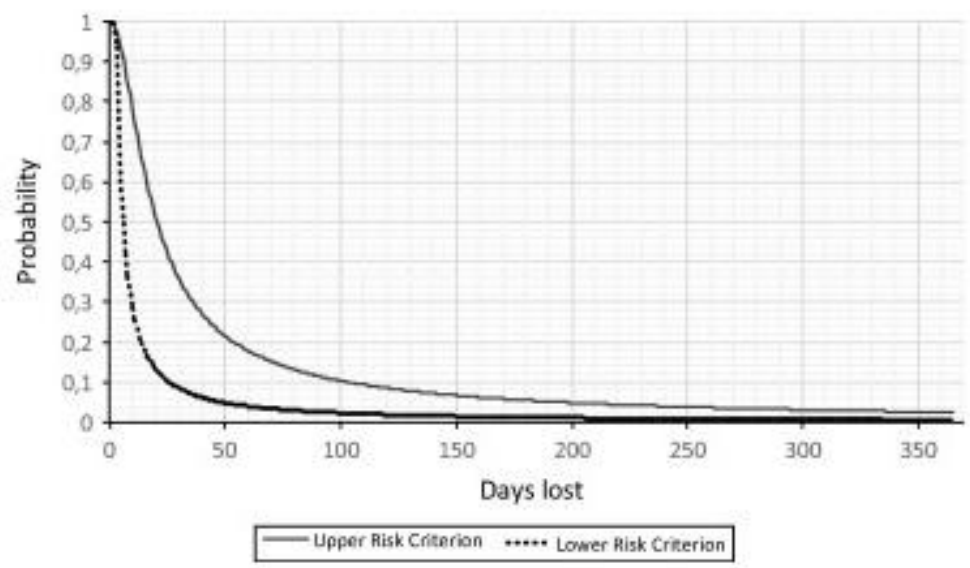

Fig. 5 .

Acceptance criteria to apply in furniture industry companies.

In the final decision regarding acceptance criteria, judgements of other stakeholders were also considered. Rodrigues et al. (2015a) have analysed the acceptance level for accident risk in the furniture industry by workers, employers and supervisors. They found that only scenarios with low risk levels were classified as being acceptable. This is in line with the results found for OSH practitioners and included in the proposed acceptance criteria. Rodrigues et al. (2015a) also found the stakeholders to assess death scenarios as being completely unacceptable, even with a notably low probability. In the light of this assumption, additional criteria to those already presented in Fig. 5 are needed, i.e. any risk that can result in a fatality must be classified as unacceptable. This is the most obvious difference between the criteria defined in this study and the acceptance criteria included in various risk assessment methodologies. For example, Woodruff (2005), in his proposed semi-quantitative methodology for application in lower hazard industrial and commercial sectors, considered the acceptance criteria presented in HSE (2001) for deaths. According to HSE (2001), the upper limit for individual risks is one fatality in 1000 persons per year, and the lower limit is one fatality in 1,000,000 persons per year. Applying these criteria to the case of the furniture sector in Portugal and considering the accident statistics, this 
would mean that it is "tolerable" to record fewer than 32 deaths per year and that it is broadly "acceptable" to record less than one death in approximately 31 years in this sector. Looking at these numbers, it is clear that these criteria cannot be considered appropriate for this sector, where in general only one death occurs per year (based on the dataset provided by GEP for this study for the years 2006-2010). Moreover, it is important to note that, in general, the criteria defined in this study are more stringent than the criteria included in risk assessment methodologies also applied to occupational accidents and using a similar risk matrix (see e.g. Marhavilas and Koulouriotis, 2008 and Jacinto and Silva, 2010). These findings support the need to use a systematic process, based on scientific approaches, to define the acceptance criteria in order to make them accurate, practical, credible and equitable (CCPS, 2009).

By applying the current criteria, three risk regions were established: unacceptable, tolerable and acceptable. The ALARP principle is applied to the risk levels that fall between both thresholds according to the TOR framework (HSE, 2001).

According to ISO 31000:2009, risk acceptance criteria must be adjusted to the companies' own safety cultures, which makes them more practicable and appropriate to the companies' specific needs. Accordingly, the proposed criteria must be considered a starting point for the criteria to be used by the companies. As long as the companies' safety cultures increase, the thresholds must be adjusted to make the severity of all accident occurrences close to zero lost workdays. This is an important step in the sense that companies with a better safety culture have a better safety performance, and, accordingly, a lower number and severity of accidents are expected (Nielsen et al., 2008, Tharaldsen et al., 2008, Vinodkumar and Bhasi, 2009, Lu and Yang, 2011 and Fugas et al., 2012).

The adjustment of acceptance criteria to companies' safety cultures can be achieved by progressively adjusting the Burr distribution parameters and consequently adjusting the CDF curve to the data obtained. For example, Fig. 6 shows the risk acceptance thresholds that were adjusted to a specific company with a high safety culture level. The initial Burr distribution parameters were adjusted using a multiplicative factor of 0.8 for the maximum tolerable risk level $(k=0.4149, \alpha=2.6353, \beta=10.1056)$ and 0.15 for the acceptable threshold ( $k=0.0778, \alpha=14.0553, \beta=1.8948)$. Therefore, the acceptance criteria have become stricter. 


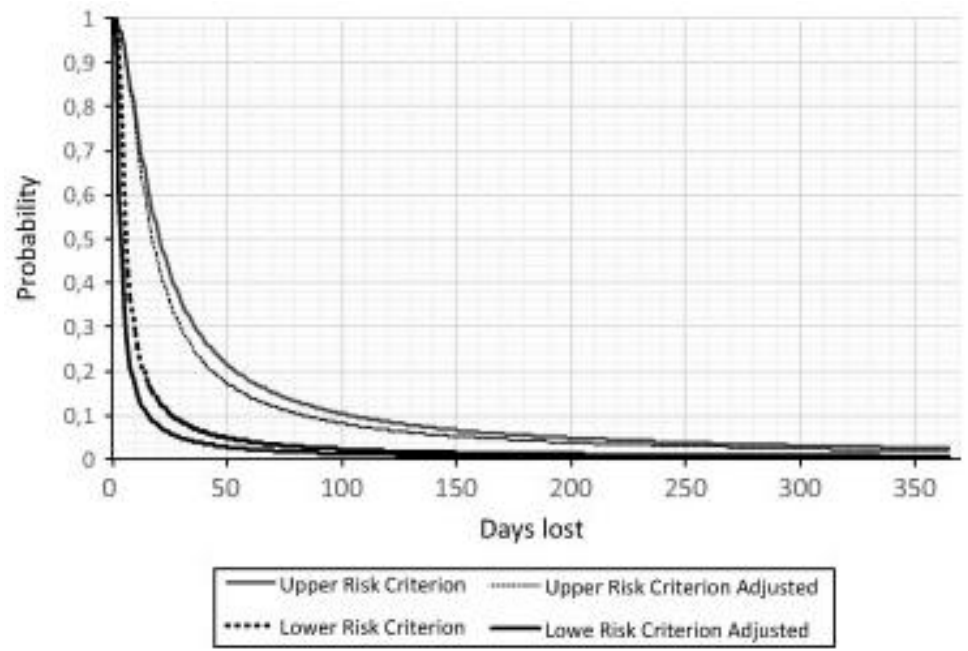

Fig. 6 .

Example of the acceptance criteria adjusted to a company with a high safety culture level.

To demonstrate the integration of the acceptance criteria into a risk metric, an example of a risk matrix developed with the data obtained is shown in Table 1. The matrix proposed in this study presents the percentage of accident frequency and corresponding severity that refers to more lost days than a given magnitude in the same way as the risk matrix proposed by Jacinto and Silva (2010). Seven severity classes were defined. In the work of Marhavilas and Koulouriotis (2008), the references of three days lost and three weeks (21 days) of absence from work were used for the severity. These values were also considered in this study. However, because the presented thresholds fall sharply between three days and three weeks, one week of absence from work (seven days) was also included in the risk matrix. Both three and six months (90 and 180 days, respectively) were also used as references to define the severity classes. The rules for risk acceptance for each combination of frequency and severity categories were defined considering the criteria in Fig. 5. For each class of severity, the frequency can change considerably in some cases; thus, the lowest corresponding limit of the interval was used. 


\begin{tabular}{|c|c|c|c|c|c|c|c|}
\hline \multirow{3}{*}{$\begin{array}{l}\text { Frequency of } \\
\text { accidents (\%) }\end{array}$} & \multicolumn{7}{|c|}{ Severity } \\
\hline & \multicolumn{6}{|c|}{ Lost workdays } & \multirow[t]{2}{*}{ Death } \\
\hline & {$[0-3]$} & {$[3-7 \mid$} & [7-21] & [21-90[ & [90-180] & $\geqslant 180$ & \\
\hline [90-100] & $\mathbf{T}$ & U & $\mathrm{U}$ & $\mathrm{U}$ & U & U & U \\
\hline ]80-90] & $\mathrm{T}$ & $\mathrm{T}$ & $\mathbf{U}$ & $U$ & $\mathbf{U}$ & U & $\mathbf{U}$ \\
\hline $\mid 70-80]$ & $\mathbf{T}$ & $\mathrm{T}$ & $\mathrm{U}$ & $\mathrm{U}$ & U & $\mathbf{U}$ & $\mathbf{U}$ \\
\hline j60-70 & A & $\mathrm{T}$ & $\mathrm{U}$ & $\mathrm{U}$ & U & U & U \\
\hline ]50-60] & A & $\mathrm{T}$ & $\mathrm{u}$ & U & U & U & U \\
\hline$|40-50|$ & A & $\mathrm{T}$ & $\mathbf{T}$ & $\mathrm{U}$ & $\mathrm{U}$ & $\mathrm{U}$ & $\mathbf{U}$ \\
\hline$[30-40]$ & A & A & $\mathbf{T}$ & U & U & U & U \\
\hline$|20-30|$ & A & A & $\mathbf{T}$ & $U$ & $\mathrm{U}$ & U & $\mathbf{U}$ \\
\hline ]10-20] & A & A & $\mathrm{T}$ & U & U & U & U \\
\hline [5-10] & A & A & A & $\mathrm{T}$ & U & $\mathbf{U}$ & $\mathbf{U}$ \\
\hline$[1-5]$ & A & A & A & $\mathrm{T}$ & $\mathrm{T}$ & $\mathrm{U}$ & $\mathrm{U}$ \\
\hline ]0.5-1] & A & A & A & A & A & $\mathrm{T}$ & U \\
\hline
\end{tabular}

To exemplify its application, a specific case of a company that recorded 10 accidents in the last year was considered. Eight of these accidents resulted in one lost workday, one accident resulted in 16 lost workdays, and one accident resulted in 90 lost workdays. The most severe situation was related to unsafe behaviours, i.e. removing pieces that blocked the machines by hand without first stopping the machine. Because one accident in this company corresponds to $10 \%$ of the total number of accidents, from the matrix, 90 lost workdays belongs to the fifth severity category, where the risk is considered to be "Unacceptable." Therefore, risk reduction measures need to be applied.

Using the proposed risk matrix, the aggregated data of the companies or the sector can be used to support decisions regarding the risk of occupational accidents and respond to new approaches suggested to this area in order to reduce subjectivity in decisions about risk (see, e.g., Jacinto and Silva, 2010 and Carrillo-Castrillo et al., 2014). Further, the presented approach has the advantage of using the frequency of accidents as a percentage. Therefore, it can be applied to all of the companies in the sector, which have several differences in size and safety performance (Rodrigues et al., 2015b).

Despite the importance of the proposed acceptance criteria in supporting the decision making regarding the treatment of risk and setting priorities, it is important to keep in mind that no limit for the number of the companies' accidents was established in this study. Therefore, each company needs to define its own additional criteria in subsequent work. Furthermore, it is not enough to just consider the risk limits to support risk decisions. As previously mentioned, a number of other factors are also important and cannot be disregarded, such as legal requirements, company policies and rules, and good practices, among others (HSE, 2001, Abrahamsen and Aven, 2008 and Harms-Ringdahl, 2013). 


\section{Conclusions}

This paper has focused on the problem of defining quantitative risk acceptance criteria. The main difficulties were mentioned, and the steps in this process regarding occupational settings were described.

The conducted work showed that, in general, the steps presented can be used to define practical, accurate and ethical acceptance criteria and are a valuable input to the overall risk assessment process. One important step in this study was the selection of the principle/philosophy to consider when defining acceptance criteria, as this can affect all of the remaining steps. Considering the ALARP principle has led to the need to define two acceptance criteria, a maximum tolerable risk level and an acceptable risk level.

It is also important to note the relevance attributed in this study to accident statistics and the judgements of OSH practitioners in the acceptance criteria definition process. Although all of the stakeholders' judgements were considered, OSH practitioners have greater knowledge about the risk assessment process and the occurrence of accidents, therefore their judgements proved to be highly important in this process. It was observed that they consider the scenarios above the sector's accident distribution to be unacceptable, which enables this boundary to be considered as the upper risk criterion.

Another important aspect of the adopted procedure was the consideration of the companies' safety cultures when defining the corresponding acceptance criteria. In fact, it is difficult to use the same acceptance criteria for all companies, even if they belong to the same sector. In this context, and assuming that the safety culture is a good indicator of the companies' safety performance and may have an influence on the stakeholders' views, it was used as a reference to adjust the acceptance criteria. This adjustment results in stricter acceptance criteria when the level of a company's safety culture is high.

The procedure proposed in this study will hopefully be useful in overcoming various practical difficulties faced by the authorities, companies and experts when formulating risk acceptance criteria, resulting in a more accurate and useful process.

\section{References}

E.B. Abrahamsen, T. Aven On the consistency of risk acceptance criteria with normative theories for decision-making Reliab. Eng. Syst. Saf., 93 (12) (2008), pp. 19061910

A.Y. Al-Saiari, L.A. Baharith, S.A. Mousa Marshall-Olkin extended Burr Type XII distribution Int. J. Stat. Probab., 3 (1) (2014), pp. 78-84 
A. Boholm

Comparative studies of risk perception: a review of twenty years of research

J. Risk Res., 1 (2) (1998), pp. 135-163

M. Burgman, F. Fidler, M. McBride, T. Walshe, B. Wintle Eliciting Expert Judgments: Literature Review Australian Centre for Excellence in Risk Analysis (ACERA), Australia (2006)

I. Cameron, R. Raman Process Systems Risk Management Elsevier, San Diego, USA (2005)

J.A. Carrillo-Castrillo, J.C. Rubio-Romero, J. Guadix, L. Onievac Risk assessment of maintenance operations: the analysis of performing task and accident mechanism Int. J. Injury Control Saf. Promot. (2014) http://dx.doi.org/10.1080/17457300.2014.939196

Center for Chemical Process Safety (CCPS) Guidelines for Developing Quantitative Safety Risk Criteria John Wiley \& Sons, New Jersey, USA (2009)

P.J. Coleman, J.C. Kerkering Measuring mining safety with injury statistics: lost workdays as indicators of risk J. Saf. Res., 38 (5) (2007), pp. 523-533

X. Cuny, M. Lejeune Occupational risks and the value and modelling of a measurement of severity Saf. Sci., 31 (3) (1999), pp. 213-229

X. Cuny, M. Lejeune Statistical modelling and risk assessment Saf. Sci., 41 (1) (2003), pp. 29-51

Eurostat European Statistics on Accidents at Work (ESAW) - Summary Methodology Publications Office of the European Union, Luxemburg (2013) (2013 edit)

M. Fera, R. Macchiaroli Appraisal of a new risk assessment model for SME Saf. Sci., 48 (10) (2010), pp. 1361-1368

C.S. Fugas, S.A. Silva, J.L. Melia Another look at safety climate and safety behavior: deepening the cognitive and social mediator mechanisms Accident. Anal. Prev., 45 (2012), pp. 468-477

L. Harms-Ringdahl Guide to Safety Analysis for Accident Prevention IRS Riskhantering AB, Sweden (2013) 
D. Hartford Legal framework considerations in the development of risk acceptance criteria Struct. Saf., 31 (2) (2009), pp. 118-123

Health and Safety Executive (HSE) Reducing Risks, Protecting People. HSE's Decision Making Process HSE Books, London (2001)

N.M.A. Huijts, E.J.E. Molina, L. Steg Psychological factors influencing sustainable energy technology acceptance: a review-based comprehensive framework Renew. Sustain. Energy Rev., 16 (1) (2012), pp. 525-531

ISO 31000:2009. Risk management - principles and guidelines. International Organization for Standardization.

ISO 31010:2009. Risk management - risk assessment techniques. International Organization for Standardization.

ISO Guide 73:2002. Risk management-vocabulary-guidelines for use in standards. International Organization for Standardization.

C. Jacinto, C. Silva A semi-quantitative assessment of occupational risks using bow-tie representation Saf. Sci., 48 (8) (2010), pp. 973-979

I.L. Johansen, M. Rausand Foundations and choice of risk metrics Saf. Sci., 62 (2014), pp. 386-399

U. Kjellén, S. Sklet Integrating analyzes of the risk of occupational accidents into the design process. Part I: A review of types of acceptance criteria and risk analysis methods Saf. Sci., 18 (3) (1995), pp. 215-227

M.L.P. Lima Percepção de riscos ambientais L. Soczka (Ed.), Contextos humanos e psicologia ambiental, Fundação Calouste Gukbenkian, Lisboa, Portugal (2005)

C.-S. Lu, C.-S. Yang Safety climate and safety behavior in the passenger ferry context Accident Anal. Prevent., 43 (1) (2011), pp. 329-341

F. Manuele, B. Main On acceptable risk Occupational Hazards (2002) 
P.K. Marhavilas, D. Koulouriotis, V. Gemeni Risk analysis and assessment methodologies in the work sites: on a review, classification and comparative study of the scientific literature of the period 2000-2009 J. Loss Prev. Process Ind., 24 (5) (2011), pp. 477-523

P.K. Marhavilas, D.E. Koulouriotis A risk-estimation methodological framework using quantitative assessment techniques and real accidents' data: application in an aluminum extrusion industry J. Loss Prev. Process Ind., 21 (6) (2008), pp. 596-603

A.S. Miguel, G. Perestrelo, J.M. Machado, M. Freitas, F. Campelo, et al. Manual de segurança higiene e saúde no trabalho para as industrias da fileira da madeira Associação das Indústrias de Madeira e Mobiliário de Portugal (AIMMP), Porto (2005)

K.M. Mohan, J.M. Miller, V. Dobson, E.M. Harvey, D.L. Sherrill Inter-rater and intrarater reliability in the interpretation of MTI photoscreener photographs of Native American Preschool Children

K.J. Nielsen, K. Rasmussen, D. Glasscock, S. Spangenberg Changes in safety climate and accidents at two identical manufacturing plants Saf. Sci., 46 (3) (2008), pp. 440-449

E. Papadimitriou, A. Theofilatos, G. Yannis Patterns of pedestrian attitudes, perceptions and behaviour in Europe Saf. Sci., 53 (2013), pp. 114-122

N. Pidgeon Risk assessment, risk values and the social science programme: why we do need risk perception research Reliab. Eng. Syst. Saf., 59 (1) (1998), pp. 5-15

A. Pinto, R.A. Ribeiro, I.L. Nunes Fuzzy approach for reducing subjectivity in estimating occupational accident severity Accid. Anal. Prev., 45 (2012), pp. 281-290

O. Renn The role of risk perception for risk management Reliab. Eng. Syst. Saf., 59 (1) (1997), pp. 49-62

Rodrigues, M.A., Arezes, P.M., Leão, C.P., 2012. Risk decision: main constraints and approaches. In: ASME 2012 International Mechanical Engineering Congress and Exposition, Design, Materials and Manufacturing, Parts A, B, and C, November 9-15, Houston, Texas, USA, vol. 3, pp. 1757-1763, ISBN: 978-0-7918-4519-6.

M.A. Rodrigues, P. Arezes, C.P. Leão Risk acceptance in the furniture sector: analysis of acceptance level and relevant influence factors Human Ecol. Risk Assess.: Int. J., 21 (5) (2015), pp. 1361-1378 
M.A. Rodrigues, P. Arezes, C.P. Leão Safety climate and its relationship with furniture companies' safety performance and workers' risk acceptance Theor. Issues Ergonom. Sci., 16 (4) (2015), pp. 412-428

M. Sari, A.S. Selcuk, C. Karpuz, H.S.B. Duzgun Stochastic modeling of accident risks associated with an underground coal mine in Turkey Saf. Sci., 47 (1) (2009), pp. 78-87

S.M. Satish, S. Bharadhwaj Information search behaviour among new car buyers: a twostep cluster analysis IIMB Manage. Rev., 22 (1-2) (2010), pp. 5-15

M.-Y. Shih, J.-W. Jheng, L.-F. Lai A two-step method for clustering mixed categroical and numeric data Tamkang J. Sci. Eng., 13 (1) (2010), pp. 11-19

M. Siegrist, G. Cvetkovich Perception of hazards: the role of social trust and knowledge Risk Anal., 20 (5) (2000), pp. 713-719

J.E. Tharaldsen, E. Olsen, T. Rundmo A longitudinal study of safety climate on the Norwegian continental shelf Saf. Sci., 46 (3) (2008), pp. 427-439

F.H.van van Duijne, D.van Aken, E.G. Schouten Considerations in developing complete and quantified methods for risk assessment Saf. Sci., 46 (2) (2008), pp. 245-254

E. Vanem Ethics and fundamental principles of risk acceptance criteria Saf. Sci., 50 (4) (2012), pp. 958-967

M.N. Vinodkumar, M. Bhasi Safety climate factors and its relationship with accidents and personal attributes in the chemical industry Saf. Sci., 47 (5) (2009), pp. 659-667

J.M. Woodruff Consequence and likelihood in risk estimation: a matter of balance in UK health and safety risk assessment practice Saf. Sci., 43 (5-8) (2005), pp. 345-353 